\title{
Materiales en lámina delgada sobre sustratos cerámicos para aplicaciones fotovoltaicas
}

\author{
C. GUILLÉN' ${ }^{1}$ J. HERRERO', M. T. GUTIÉRREZ' Y F. LUCAS ${ }^{2}$ \\ ${ }^{1}$ Departamento de Energías Renovables (CIEMAT), Avda. Complutense 22, Madrid 28040. \\ ${ }^{2}$ Fritta, S. L., Ctra. CV-20 Km 8, Onda (Castellón) 12200.
}

\begin{abstract}
Se han probado distintos tipos de soportes cerámicos y vidrio sodocálcico convencional como sustratos sobre los que se han depositado películas de molibdeno y seleniuro de cobre e indio (heteroestructuras de la forma sustrato/ $\mathrm{Mo} / \mathrm{CuInSe}_{2}$ ) para su aplicación en células solares fotovoltaicas de lámina delgada. Las películas de Mo se han preparado simultáneamente sobre los distintos sustratos mediante evaporación asistida por cañón de electrones. A continuación, las láminas delgadas de CuInSe ${ }_{2}$ se han obtenido mediante electrodepósito directo sobre los sustratos cubiertos con Mo. Se ha determinado la influencia del tipo de sustrato empleado sobre las propiedades ópticas y estructurales de los materiales preparados, con objeto de evaluar las posibilidades de sustitución del soporte de vidrio usualmente utilizado por materiales cerámicos que permitan diversificar las aplicaciones fotovoltaicas de este tipo de sistemas. Los resultados obtenidos indican que el factor de dispersión y la tensión estructural de las láminas desarrolladas se minimizan cuando la morfología superficial del soporte cerámico se aproxima a la del vidrio convencional.
\end{abstract}

Palabras clave: $\mathrm{CuInSe} \mathrm{I}_{2} / \mathrm{Mo}$, láminas delgadas, reflectancia, células solares fotovoltaicas.

\section{Thin film materials on ceramic substrates for photovoltaic applications}

Various ceramic supports besides standard soda-lime glass have been utilized as substrates for deposition of molybdenum and copper indium diselenide layers (substrate/Mo/CuInSe $\mathrm{H}_{2}$ heterostructures) for application in thin film photovoltaic solar cells. Molybdenum layers have been prepared simultaneously onto the different substrates by electron gun assisted evaporation. Subsequently, CuInSe ${ }_{2}$ thin films have been obtained by direct electrodeposition onto the Mo-coated substrates. The influence of the substrate characteristics onto the optical and structural properties of the developed materials has been determined to evaluate the possibility of substituting the commonly utilized glass by ceramics that can wide the applications of these photovoltaic systems. The obtained results indicate that the scattering factor and the structural stress in the layers are minimized when the morphological characteristics of the ceramic are close to those of the standard glass substrates.

Key words: CuInSe, Mo, thin films, reflectance, photovoltaic solar cells.

\section{INTRODUCCIÓN}

Los dispositivos fotovoltaicos de lámina delgada con absorbentes del tipo calcopirita basados en el seleniuro de cobre e indio han alcanzado ya altos valores de eficiencia de conversión de la energía solar en energía eléctrica, y en un futuro próximo se espera alcanzar su producción a escala industrial para la obtención de electricidad $(1,2)$. La fabricación de dichos dispositivos fotovoltaicos se realiza mediante la preparación de distintas láminas delgadas de metales y semiconductores, apiladas sobre un sustrato que normalmente es vidrio sodocálcico convencional (2). Existe un creciente interés en el desarrollo de los dispositivos sobre sustratos alternativos al vidrio, con el objetivo de diversificar las aplicaciones y usos de esta tecnología (3-5). Los materiales cerámicos se presentan como una interesante propuesta, cuya validez vendrá determinada por la posibilidad de mantener la calidad fotovoltaica de los materiales depositados sobre estos nuevos sustratos.

El análisis óptico y estructural del sistema formado por películas de molibdeno y seleniuro de cobre e indio depositadas sobre el sustrato seleccionado, heteroestructuras de la forma sustrato/Mo/CuInSe, ${ }_{2}$ se ha mostrado de gran importancia para el desarrollo de células solares de lámina delgada de alta eficiencia (6). En general, resulta necesario minimizar el factor de dispersión, la rugosidad superficial y la tensión estructural de la película de CuInSe ${ }_{2}$ para hacer máxima la absorción, mínima la densidad de defectos y mejorar la calidad final de la célula solar. En este sentido, es importante tener en cuenta que las propiedades de estos materiales crecidos en lámina delgada sobre distintos sustratos son diferentes de las de los materiales en volumen, debido a sus características especiales de microestructura y densidad, la gran proporción de superficie respecto del volumen y otros factores impuestos por las particularidades del sustrato utilizado.

En el presente trabajo se han probado distintos tipos de soportes cerámicos además de vidrio sodocálcico convencional para desarrollar heteroestructuras de la forma sustrato/Mo/CuInSe ${ }_{2}$, depositando sobre los diferentes sustratos láminas delgadas de Mo mediante evaporación asistida por cañón de electrones, y utilizando dichos sustratos recubiertos 
con molibdeno como electrodos para la obtención de películas de CuInSe ${ }_{2}$ mediante electrodepósito directo. Se han realizado análisis óptico y estructural de las muestras preparadas, para determinar la influencia del sustrato utilizado sobre la reflectancia óptica, el factor de dispersión, la orientación preferencial y la tensión estructural de las láminas de Mo y de CuInSe ${ }_{2}$ desarrolladas para aplicaciones fotovoltaicas.

\section{PARTE EXPERIMENTAL}

Tres tipos distintos de sustratos planos cerámicos, que denominaremos $\mathrm{C} 1, \mathrm{C} 2$ y C3, se han utilizado junto con vidrio sodocálcico convencional como sustratos sobre los que se prepararon láminas delgadas de molibdeno mediante evaporación asistida por cañón de electrones. El tipo de sustrato denominado $\mathrm{C} 1$ corresponde a gres porcelánico con un esmalte de estructura mate granular, cuyos componentes mayoritarios son $\mathrm{SiO}_{2}, \mathrm{Al}_{2} \mathrm{O}_{3}, \mathrm{BaO}, \mathrm{CaO}$ y $\mathrm{ZnO}$, con un pequeño porcentaje de $\mathrm{Na}_{2} \mathrm{O}$ y K $\mathrm{K}_{2} \mathrm{O}$. El tipo $\mathrm{C} 2$ corresponde a gres porcelánico con un esmalte transparente brillante aplicado en líquido y pulido después de la cocción, de componentes fundamentales $\mathrm{SiO}_{2}$, $\mathrm{CaO}, \mathrm{Al}_{2} \mathrm{O}_{3^{\prime}}, \mathrm{ZnO}$ y K $\mathrm{K}_{2} \mathrm{O}$. El tipo $\mathrm{C} 3$ corresponde a gres porcelánico con un esmalte transparente brillante aplicado en seco y pulido después de la cocción, de componentes fundamentales $\mathrm{SiO}_{2}, \mathrm{Al}_{2} \mathrm{O}_{3}, \mathrm{ZnO}, \mathrm{CaO}, \mathrm{K}_{2} \mathrm{O}, \mathrm{BaO}, \mathrm{B}_{2} \mathrm{O}_{3}$ y $\mathrm{MgO}$. La evaporación de las láminas de molibdeno sobre los distintos sustratos se llevó a cabo en el sistema experimental descrito en anteriores trabajos (7), manteniendo una velocidad de crecimiento constante de $10 \AA / \mathrm{s}$, hasta alcanzar un espesor final de la lámina de Mo de aproximadamente $1 \mu \mathrm{m}$. A continuación, se prepararon películas de $\mathrm{CuInSe}_{2}$ mediante electrodepósito directo sobre los sustratos cubiertos con Mo evaporado. El proceso de electrodepósito consiste en la reducción simultánea de

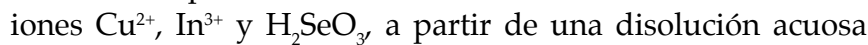
conteniendo sulfatos de cobre y de indio, con ácido de selenio (8), sobre el sustrato al que se aplica un potencial adecuado, ajustando el potencial y el tiempo de depósito para obtener láminas de composición estequiométrica de $2 \mu \mathrm{m}$ de espesor, con un ritmo de crecimiento constante de aproximadamente $12 \AA / s$. Todos los procesos de preparación se realizaron a temperatura ambiente.

Las medidas ópticas de las láminas de Mo y de CuInSe $e_{2}$ depositadas sobre los distintos sustratos se han realizado en el intervalo de longitudes de onda desde 400 hasta $1500 \mathrm{~nm}$ con un espectrofotómetro Cary 5000 que incluye una esfera integradora para la determinación de la reflectancia difusa y la reflectancia total de las muestras. Las características de rugosidad superficial de las láminas se han obtenido mediante un perfilómetro Dektak. Asimismo se han llevado a cabo medidas de difracción de rayos $\mathrm{X}(\mathrm{XRD})$ en un difractómetro Philips X'pert, utilizando la radiación $\mathrm{K}_{\alpha 1}$ del $\mathrm{Cu}$, que han permitido determinar la orientación cristalográfica preferencial y la tensión estructural en las láminas delgadas de Mo y de CuInSe ${ }_{2}$ preparadas.

\section{RESULTADOS Y DISCUSIÓN}

Los valores de reflectancia total $\left(R_{t}\right)$ y reflectancia difusa $\left(\mathrm{R}_{\mathrm{d}}\right)$ obtenidos para las láminas de molibdeno evaporadas simultáneamente sobre distintos sustratos se muestran en la Figura 1, donde puede observarse que dichos valores, espe-

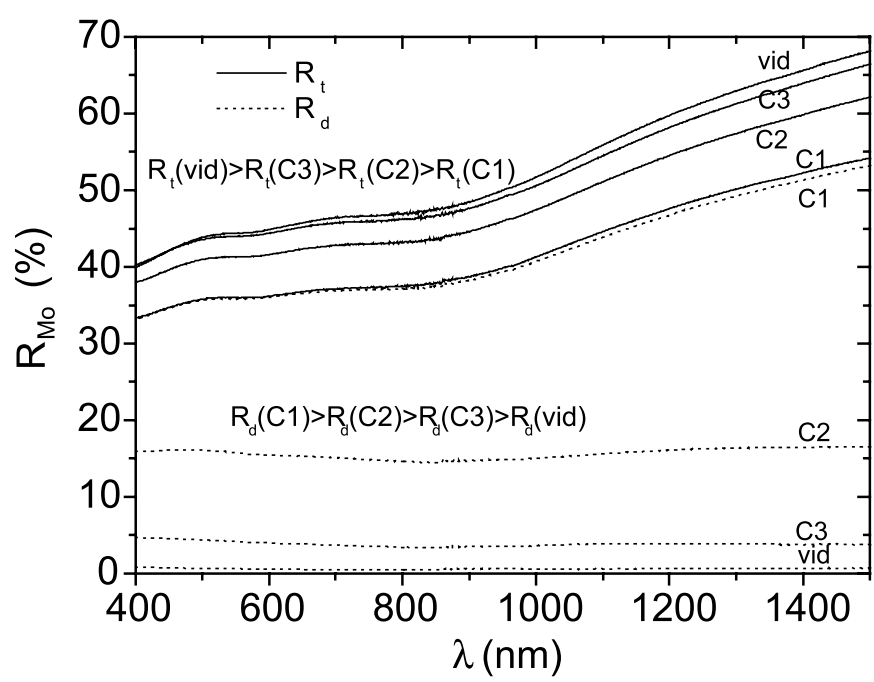

Figura 1: Espectros de reflectancia total $\left(\mathrm{R}_{\mathrm{t}}\right)$ y reflectancia difusa $\left(\mathrm{R}_{\mathrm{d}}\right)$ correspondientes a láminas de Mo depositadas sobre distintos tipos de sustratos cerámicos, denominados $\mathrm{C} 1, \mathrm{C} 2$ y C3, y sobre vidrio sodocálcico.

cialmente los de reflectancia difusa, resultan fuertemente dependientes del sustrato utilizado. En las láminas de Mo el aumento de la reflectancia total en la zona espectral del infrarrojo se ha relacionado con un aumento de la conductividad eléctrica del material (7). Por otra parte, el cociente entre los valores de reflectancia difusa y reflectancia total nos da el factor de dispersión óptica de las láminas $\left(F D=R_{d} / R_{t}\right)$, calculado en la Tabla I, que está relacionado con la rugosidad superficial del material depositado, cuyo valor determinado mediante perfilometría se ha representado en la misma tabla. La calidad de la película delgada de Mo para su aplicación como contacto eléctrico dorsal en dispositivos fotovoltaicos mejora cuando aumentan la reflectancia total y la conductividad eléctrica, y cuando disminuyen el factor de dispersión y la rugosidad superficial. Los datos ópticos obtenidos indican que la reflectancia total es máxima y el factor de dispersión mínimo, según las consideraciones anteriores la calidad es máxima, para las láminas de Mo evaporadas sobre vidrio.

TABLA I: VALORES DEL FACTOR DE DISPERSIÓN (FD) Y LA RUGOSIDAD SUPERFICIAL ( $\mathrm{R}_{\mathrm{A}}$ ) OBTENIDOS PARA LAS LÁMINAS DE MO Y DE CUINSE ${ }_{2}$ (CIS) DEPOSITADAS SOBRE DISTINTOS SUSTRATOS.

\begin{tabular}{|c|c|c|c|c|}
\hline Sustrato & $\mathrm{FD}(\mathrm{Mo})$ & $\mathrm{R}_{\mathrm{a}}(\mathrm{Mo})(\mathrm{nm})$ & $\mathrm{FD}(\mathrm{CIS})$ & $\mathrm{R}_{\mathrm{a}}(\mathrm{CIS})(\mathrm{nm})$ \\
\hline Cerámico C1 & 0.99 & 1330 & 1.00 & 2100 \\
\hline Cerámico C2 & 0.42 & 125 & 0.94 & 308 \\
\hline Cerámico C3 & 0.12 & 35 & 0.96 & 318 \\
\hline Vidrio & 0.02 & 4 & 0.67 & 220 \\
\hline
\end{tabular}

Las características ópticas de las láminas de $\mathrm{CuInSe}_{2}$ depositadas sobre los distintos sustratos recubiertos con Mo se muestran en la Figura 2 y también en la Tabla I, junto a los datos correspondientes a su rugosidad superficial. En el caso de estas láminas que actuarán como absorbente en el dispositivo fotovoltaico, la disminución de los valores de reflectancia a longitudes de onda inferiores a unos $1200 \mathrm{~nm}$, correspondiendo a energías de la radiación superiores a 1.0 eV, es debida al aumento del coeficiente de absorción para energías superiores 


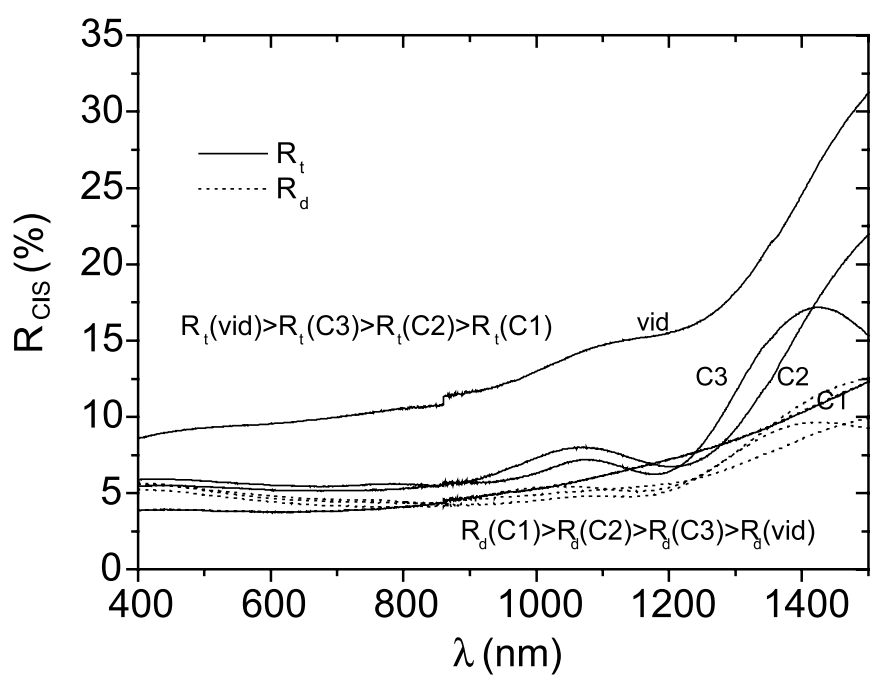

Figura 2: Espectros de reflectancia total $\left(R_{t}\right)$ y reflectancia difusa $\left(R_{d}\right)$ correspondientes a láminas delgadas de CuInSe ${ }_{2}$ (CIS) depositadas sobre los distintos sustratos cubiertos con Mo.

a la anchura de la banda prohibida del semiconductor. Los resultados obtenidos indican que las láminas de $\mathrm{CuInSe}_{2}$ (CIS) depositadas sobre los cerámicos $\mathrm{C} 2$ y C3 presentan una variación óptica espectral y un valor de rugosidad superficial que se aproximan a los obtenidos sobre vidrio. Los datos de la tabla I muestran que el factor de dispersión de las láminas de CuInSe ${ }_{2}$ es siempre superior al obtenido para las láminas de Mo sobre el mismo sustrato, lo que está relacionado con la mayor rugosidad de la superficie de CuInSe ${ }_{2}$ que de la capa de Mo, rugosidad que es aún mayor sobre los soportes cerámicos que sobre el vidrio sodocálcico.

En la figura 3 se han representado los diagramas de difracción de rayos $\mathrm{X}$ obtenidos para los materiales en lámina delgada desarrollados sobre los distintos sustratos. En dichos diagramas se identificaron como picos de difracción principales los correspondientes a los planos cristalográficos (110) del Mo de estructura cúbica (9) y a los planos (112) del CuInSe de estructura tetragonal (10). La posición angular de estos picos de difracción principales se ha encontrado dependiente del tipo de sustrato utilizado. En la figura 4 puede observarse que la posición del pico de difracción (110) del Mo se desplaza hacia ángulos menores, lo que indica un aumento del espaciado interplanar correspondiente, cuando se sustituye el vidrio por un sustrato cerámico. Este desplazamiento supone un aumento de la tensión estructural del tipo extensivo, que está relacionado con la disminución de la reflectancia total de la lámina de Mo (7) que anteriormente se había señalado. Por otra parte, en la figura 5 se observa que la posición del pico de difracción (112) del CuInSe ${ }_{2}$ experimenta un desplazamiento similar pero en sentido contrario al presentado por el pico (110) del Mo sobre el que está depositado. Este hecho indica que el aumento de la tensión estructural de tipo extensivo en la lámina de Mo supone un aumento de la tensión de tipo compresivo en la película de CuInSe ${ }_{2}$ situada sobre el Mo. La tensión estructural en la lámina de CuInSe ${ }_{2}$ electrodepositada se minimiza, es decir la distancia interplanar se aproxima al valor determinado para el material en volumen (10), cuando disminuyen el factor de dispersión y la rugosidad superficial del sustrato utilizado.

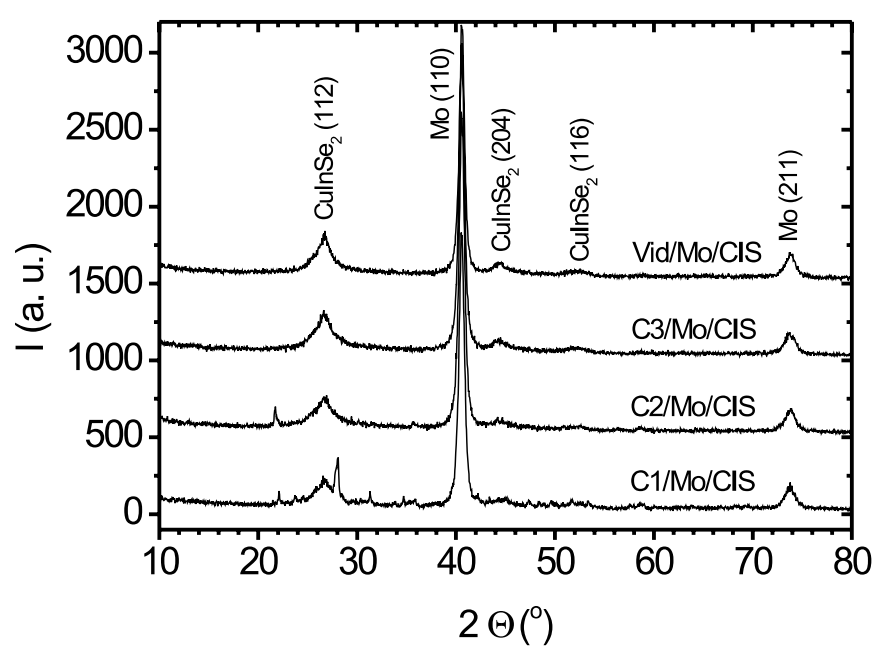

Figura 3: Diagramas de difracción de rayos $\mathrm{X}$ obtenidos para las láminas de Mo y CuInSe ${ }_{2}$ (CIS) depositadas sobre los distintos sustratos utilizados.

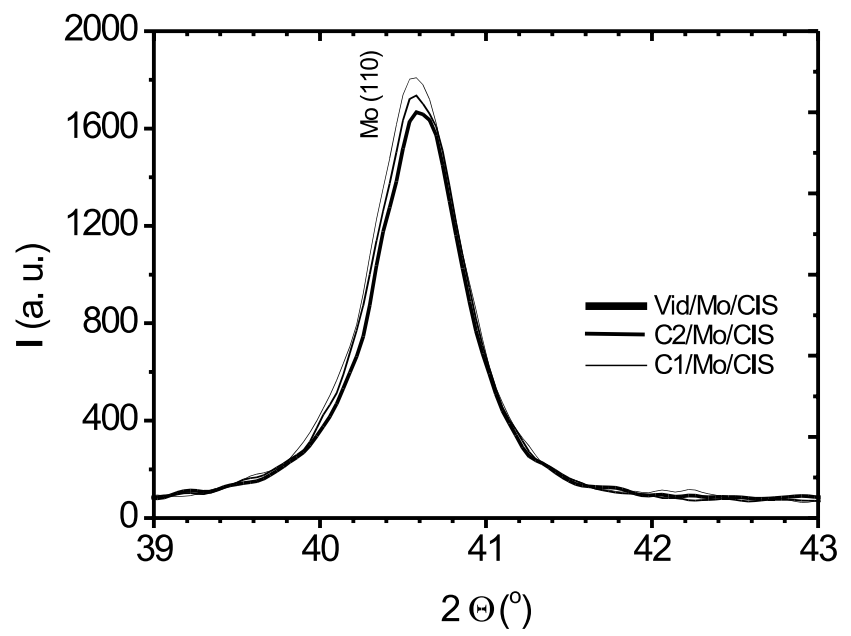

Figura 4: Detalle del pico de difracción correspondiente al Mo depositado sobre el sustrato de vidrio y sobre distintos cerámicos.

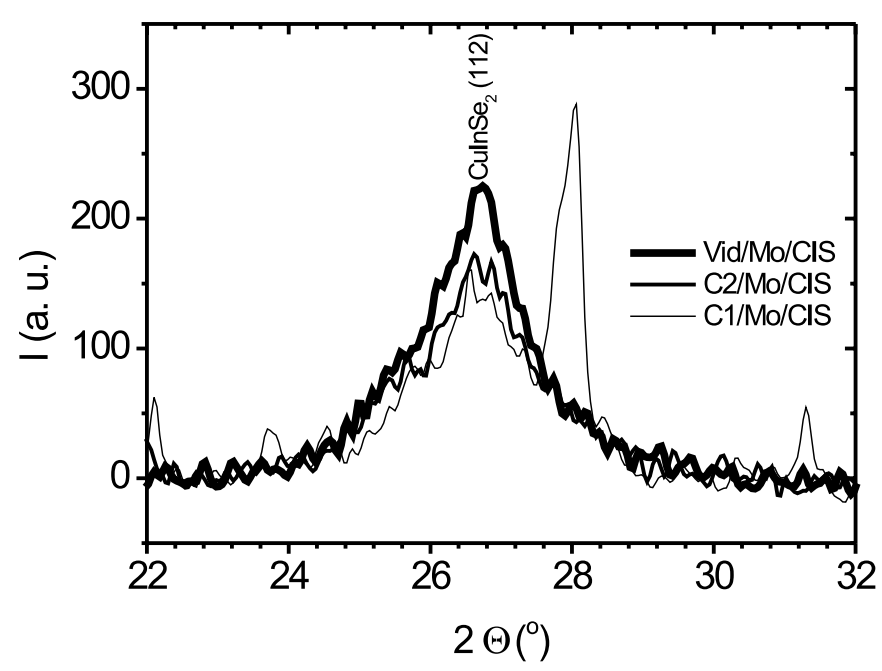

Figura 5: Detalle del pico de difracción correspondiente al CuInSe ${ }_{2}$ (CIS) depositado sobre distintos sustratos cubiertos con Mo. 


\section{CONCLUSIONES}

Mediante la evaporación de láminas delgadas de molibdeno y el posterior electrodepósito de películas de seleniuro de cobre e indio se han desarrollado estructuras de aplicación fotovoltaica sobre sustratos cerámicos alternativos al vidrio sodocálcico. El análisis óptico, morfológico y estructural de las muestras preparadas indica que el factor de dispersión y la rugosidad superficial de la lámina de Mo aumentan, a la vez que su reflectancia total disminuye, cuando el vidrio se sustituye por cualquiera de los cerámicos utilizados. Asimismo la tensión estructural en la lámina de CuInSe ${ }_{2}$ electrodepositada aumenta cuando lo hacen el factor de dispersión y la rugosidad superficial del sustrato. Para optimizar la calidad de las láminas delgadas sobre sustratos cerámicos para aplicaciones fotovoltaicas resulta necesario utilizar cerámicos de morfología superficial similar a la del vidrio convencional.

\section{AGRADECIMIENTOS}

El presente trabajo se ha realizado dentro de los proyectos "Desarrollo de materiales y dispositivos fotovoltaicos" financiado por el CIEMAT, y "Materials Research and Innovation in Solar Cells (MARISOL)" financiado por la Comunidad de Madrid.

\section{BIBLIOGRAFÍA}

1. M. A. Green, K. Emery, D. L. King, S. Igari, W. Warta. “Solar cell efficiency tables". Progress in Photovoltaics 11 [5] 347-352 (2003).

2. M. Powalla, D. Hariskos, E. Lotter, M. Oertel, J. Springer, D. Stellbogen, B. Dimmler, R. Schäffler. "Large-area CIGS modules: processes and properties". Thin Solid Films 431/432 523-533 (2003).

3. I. Lauermann, I. Luck, K. Wojczykowski. "CuInS -based thin film solar cells on roof tile substrates", pp. 1163-1165 en Proceedings of the 17th European Photovoltaic Solar Energy Conference, Munich (Alemania), Octubre 2001, James \& James Publishers Ltd. 2002.

4. I. B. Hagemann. "New developments and future perspectives of the building integrated photovoltaic market", pp. 2661-2664 en Proceedings of the 17 European Photovoltaic Solar Energy Conference, Munich (Alemania), Octubre 2001, James \& James Publishers Ltd. 2002.

5. O. Perpiñan, J. Vega, R. Eyras. "Special module types for PV systems in highprofile buildings", pp. 2713-2716 en Proceedings of the $17^{\text {th }}$ European Photovoltaic Solar Energy Conference, Munich (Alemania), Octubre 2001, James \& James Publishers Ltd. 2002.

6. M. A. Contreras, K. M. Jones, L. Gedvilas, R. Matson. "Preferred orientation in polycrystalline $\mathrm{Cu}(\mathrm{In}, \mathrm{Ga}) \mathrm{Se}_{2}$ and its effect on absorber thin-films and devices", pp. $732-735$ en Proceedings of the $16^{\text {th }}$ European Photovoltaic Solar Energy Conference, Glasgow (Reino Unido), Mayo 2000, James \& James Publishers Ltd 2001.

7. C. Guillén, J. Herrero. "Low-resistivity Mo thin films prepared by evaporation onto $30 \times 30 \mathrm{~cm}^{2}$ glass substrates", pp. 575-581 en Proceedings of the International Conference on Advanced Materials and Processing Technologies, Madrid (España), Septiembre 2001, Ed. Prof. José M. Torralba 2002.

8. C. Guillén, J. Herrero. "Reaction pathways to CuInSe formation from electrodeposited precursors". J. Electrochemical Society 142 1834-1838 (1995).

9. Ficha de difracción del Mo, $\mathrm{n}^{\circ}$ 4-809 según el “Joint Committee on Powder Diffraction Standards".

10. Ficha de difracción del CuInSe ${ }_{2^{\prime}} \mathrm{n}^{\circ}$ 23-209 según el "Joint Committee on Powder Diffraction Standards".

Recibido: 12-09-03

Aceptado: 22-06-04 